\title{
Phaco - Emulsification Using Rotating Needle - A Newer Cheaper Technique
}

\author{
Mohammad Abu Yousuf Talukder* \\ Resident Surgeon, Medical College for Women \& hospital, Bangladesh
}

Received: 13 May, 2017; Accepted: 17 October, 2017; Published: 17 November, 2017

*Corresponding author: Mohammad Abu Yousuf Talukder, Resident Surgeon, Medical College for Women \& hospital, Bangladesh, Email address: drtalukderyousuf@gmail.com

\section{Abstract}

This Phaco-emulsification device is cheap to build, easy to operate, effective for treating patients with Cataract, the device will use low power; Torque using Simple DC Motor. This method of using rotating needles was used by Dr. Charles D. Kelman . I was able to overcome the problem, i.e. spinning of the lens in A/C. The device has three parts, body,tube and Yousuf's Needle. Body contains a motor and a wire connecting the power source with a foot-switch. The motor has a shaft enabling the needle to spin. The tube has a broad base attached with body and a tapered end containing Yousuf's Needle. Yousuf's Needle is made by incorporating 3-4 hypodermic needles in a silicon tube .It become easy to penetrate, generate low heat; low angular acceleration, hence does not cause undesired effect during procedures. It was tested on wood in the first stage. Following that, samples of 50 human lenses were collected after doing SICS and used by selecting them in random but purposive manner. When human lens were handled successfully, 50 goat eyes were operated. This cross- sectional study was done within a year. The device shows moderate penetrating capacity on wood. Goat's lens was successfully emulsified by this device. There was no spinning of lens causing denuding corneal endothelium, no iris injury or Posterior Capsular rupture observed. Human lenses turned into jelly-like substances quickly, where hyper mature lens need more time to emulsify. So, this device hereby developed through the research should be given chance for further improvement.
\end{abstract}

\section{Introduction}

Cataract is a leading cause of blindness worldwide. Fortunately it is curable. A patient having cataract, his/her visual acuity will be up to hand movement or perception of light and projection of rays, but after cataract surgery visual acuity will be 6/6. From very beginning cataract surgery was not so easy and safe. We had so many bad experiences regarding intra capsular cataract extraction. We have overcome this situation by introducing extra capsular cataract extraction instead of ICCE, to minimize the complications which normally occurred during intra capsular cataract extraction surgery.

Now-a- days, it is further improved to small incision cataract surgery rather than extra capsular cataract extraction. The main improvement behind SCIS was that no suture is required where extra capsular cataract extraction required stitches.

Recent advances of cataract surgery is highly sophisticated and give the person early visual outcome, with less or no astigmatism, with no hospital staying, after undergoing cataract surgery. Phaco emulsification is the ultimate choice for new generation due to very small incision cataract surgery called micro incision cataract surgery. Only 2-3 $\mathrm{mm}$ or less incision is required and in this case nucleus of lens is emulsified by a titanium needle which vibrates on an average frequency of 28,000 to 40,000 hertz, and Amplitude is $5-15$ micron due to a signal from ultra-sound phaco emulsification machine.

However, this device has some limitations in the context of the poverty and health care in under developed and developing countries, such as Bangladesh.

First of all, it is very expensive. Cost of each machine is 40,000 $-50,000$ US dollars. Moreover, we need to change cassette, needle etc time to time, prior to surgery. This requires huge financial involvement to complete an operation. So very less number of surgeons in third world country are using this device for cataract operation.

\section{History of Phaco}

Before the invention of Phaco-emulsification machine, Charles. D. Kelman (The inventor of Phaco machine) had tried to do phaco -emulsification using rotating needle.

He tried a simple rotating device, thinking that if he could drill a tooth, he could drill away a cataract .This only made the lens spin inside the animal's eye, denuding the endothelium. He then added suction and irrigation, in an attempt to keep the lens from rotating. However, this did not prevent the lens from spinning, so he introduced other instruments in an attempt to hold the lens in place while it was being drilled. This attempt was unsuccessful too. So, he took two needles rotating in opposite directions, similar to a food blender. This approach was exceedingly dangerous, since it set up eddy currents which drew the iris to the needles, causing a complete disinsertion of the iris with hemorrhage [1].

\section{Material and Method}

New machine is very ordinary but effective. It works by rotating a special type of needle (Yousuf's needle) containing three to four needles, incorporated with each other, which is 
connected with a small motor. The said Motor rotates by DC (Direct Current) whose voltage will be $1.5 \mathrm{~V}$ to $12 \mathrm{~V}$, with the rotation rate ranging from $700-1400 \mathrm{rpm}$.

The Machine has three parts - namely Body, Tube and Yousuf's Needle.

- Body contains a motor and a wire connecting the power source with a foot-switch. The motor has a shaft enabling the needle to spin.

-The tube has a broad base attached with body and a tapered end containing Yousuf's Needle.

Unlike other phaco device, this device will not produce much more temperature causing damage to the surrounding structures. So this device can be used without any sleeve! So tapered end of tube will be $2.4 \mathrm{~mm}$ in its outer diameter and inner diameter will be $2 \mathrm{~mm}$. So operation can do with only 2.8 or $3.2 \mathrm{~mm}$ clear corneal incision.

\section{Yusuf's Needle}

1. Instead of single needle I have made it by multiple needles to get the following advantages -

-Easy penetration to the lens fibers

-Quick cutting lens fibers by increasing cutting surfaces of needle -Arrangements of needles are in such a fashion that cutting surfaces will be outside the circle

2. Diameter of Yousuf's Needle is little bit higher than the existing phaco tip. So if you consider about cutting lens fibers, then my device has advantages over existing phaco device. Existing device has a sleeve. With this sleeve total diameter of phaco tip is $2 \mathrm{~mm}$ which is more than my device. Due to production of very low temperature no need of this sleeve in my device. So with more diameter of needle it will easily enter into the anterior chamber with $2.8 \mathrm{~mm}$ clear corneal tunnel.

3. Size of needle will depend upon the nature of device.

-Device without irrigation and aspiration system - in that system size of needle will be 20 gauze needles. Total diameter of needle will be $1.85 \mathrm{~mm}$.

-Device with only aspiration system - in that system size of needle will be 22 gauze needles. Total diameter of needle will be $1.45 \mathrm{~mm}$.

-Device with irrigation and aspiration system - in that system size of needle will be 23 gauze needles. Total diameter of needle will be $1.2 \mathrm{~mm}$.

4. Generate very low temperature - less than 2'C during emulsifying lens, due to sharp cutting surface and also move slowly - it generate very low temperature. See table below (Table-1)

5. Serrated Cutting Surface - This device is worked by a low rotating needle. So I can use serrated cutting surfaces instead of plane cutting surface to enhance its efficacy, although now I am using plane cutting surface and got excellent result in emulsifying lens but there is an opportunity in my hand to increase its efficacy more.

6. Conform the needle in lens fibers. (Figure 1)

\begin{tabular}{|c|c|}
\hline $\begin{array}{c}\text { Table 1: Change of temperature in the lens during the operation in a } \\
\text { small container }\end{array}$ \\
\hline Time $(\mathrm{s})$ & Temp of Lens $\left({ }^{\circ} \mathrm{C}\right)$ \\
\hline 00 & 28.3 \\
\hline 05 & 28.6 \\
\hline 10 & 28.6 \\
\hline 15 & 28.9 \\
\hline 20 & 29.2 \\
\hline 25 & 28.8 \\
\hline 30 & 28.6 \\
\hline 35 & 28.7 \\
\hline
\end{tabular}

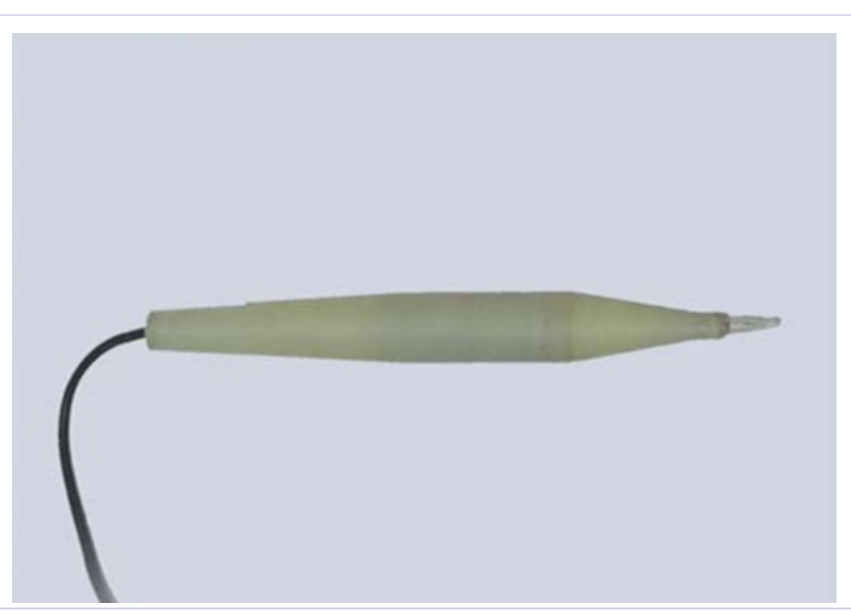

Figure 1: Phaco device using rotating needle

\section{The irrigation and aspiration systems}

An irrigation and aspiration system is very much important in case of Phaco emulsification surgery to clear up all the jelly like material which produces due to breaking up the lens matter.

On the basis of irrigation and aspiration systems, this device will be of 3 types.

\section{This device without irrigation and aspiration system}

in that case this device needs only $2 \mathrm{~mm}$ clear corneal incision for performing phaco emulsification surgery. There will be no covering of needle (Yousuf's Needle). In that case we can use two way irrigation and aspiration canola which we use for SICS (small incision cataract surgery). Obviously here we need 3 incisions. First, for device, secondly for phaco chopper and lastly for two way irrigation and aspiration canola. In that case, surgeon will hold phaco device on his/her right hand and phaco chopper on his/her left hand and assistant will hold two way irrigation and aspiration canola. So no need a sucker machine for aspiration of jelly like substances. I have used this device for all clinical trials. 


\section{This device with only aspiration system}

A modified phaco chopper (irrigating phaco chopper) will be used to hold the lens during emulsification and also irrigation done by connecting this phaco chopper with tube of balanced salt solution. A sucker machine is connected with this device. So, we can easily aspirate jelly like substances by this device. Here incision needs only $2.8 \mathrm{~mm}$ or below. Only two incisions will be needed, one for the device and other for irrigating phaco chopper.

\section{This device with irrigation and aspiration systems}

in this system, only 2 incisions needed for completing the operation, but the problem is, if we incorporate both irrigation and aspiration systems in a same tube then the diameter of tube containing Yousuf's Needle will be $3 \mathrm{~mm}$ or more. On the other hand, surgeon will hold the device on his/her right hand and phaco chopper on his/her left hand so there will be a sucker machine needed to aspirate the emulsifying material.

\section{Methods}

The following operational procedures were carried out with the proposed needle-bundle: three different ways; on wood; on goat eye; on human crystalline lens in vitro.

\section{Three different tests have been carried out successfully.}

On wood - main idea was that to observe the penetrating capacity, frequency, temperament, fluctuation of the device to get idea of differences between the wood and human crystalline lens.

Experimented on goat's eye - Second experiment was carried out with goat's eye. It is widely practiced to experiment on goat (ref no 2), sheep (ref. no. 3), or pig's (ref. No.4) eye before going experiment on human eye. Hence, this procedure was experimented on goat's eye. 50 cases were used for this clinical trial. Method was random but purposive method.

The traditional experiment runs with this device with goat's eye. After peritomy, we enter into anterior chamber by making scleral tunnel, then doing capsulotomy of anterior lens capsule, hydro dissection, and also hydro desalination was carried out. Lens was brought to anterior chamber. Then nucleus of lens of goat's eye was close to this device for emulsification of lens. After rotating of device lens turned into broken particles and afterwards became jelly like substance. Some portion of lens decayed, the process created a hole in the middle of operating portion. Afterwards the broken part of the lens was brought near the needle with the help of a phaco chopper. The device and again started to operate until, the total lens emulsified fully after a period of few minutes at a stretch.

On human crystalline lens in vitro - After operation of small incision cataract surgery, 50 lenses are collected immediately after delivery of lens and preserved it in visco- elastic substances, and balanced salt solution which is identical to aqueous humor. Lens was held with the lens holding forceps. Then this device started to rotate after penetration through lens. Three different experiments were done, after taking three different types of lenses. First was immature, another was matured and last one was hyper matured cataractous lenses. Method was random but purposive method.

All lenses were cadaveric lens which are collected after doing SICS operation.

\section{Result}

a) First experiment was done successfully on the woodPenetration of this device on wood was free and quick. Wood is a harder substance compared to human crystalline lens, so this experiment showed that easy penetration through human crystalline lens by this device will be possible. Besides this, side to side movement of rotating needle gives the idea about effective phaco emulsification by this device will be possible.

b) Second experiment with goat's eye-gives the clear cut idea about the utility of this device. Main idea is tried to evaluate how much efficiency is shown by this device, and also indicate any type of complications do occur or not? Lens of goat's eye was emulsified successfully with this device repeatedly. No spinning of the lens at all denuding corneal endothelial damage. No severe complication like posterior capsular rupture, vitrious prolaps, no corneal endothelial injury, and no wound burn occurred. Initially one minor complication was observed in anterior chamber of goat's eye, that is iris was injured by the needle but the Problem was overcome by exercising caution.

\section{c) With the human crystalline lens we observed}

\section{Three different results are as follows.}

- When experiment was carried out on immature lens, then it turned that the lenses into jelly like substances very quickly.

- When the process was applied to matured lens it has turned into jelly like substances. The procedure was in accordance with the expected rate. That is to say that the lenses have been emulsified optimally as expected.

- However, in case of hyper mature cataract, the process was time prolonged accordance with the expected rate and the lens was jelly like substance.

\section{Production of temperature}

This device produces very negligible temperature during emulsifying lens material.

\section{See table below (Table: 2,3,4) (Figure: 2,3,4)}

This data shows that very minimum change of temperatures i.e. very low Cumulative Disperse Energy (CDE) occurred when operation done by this device on both human and goat's eye. Note that fluctuation of temperature depends on density of lens, duration of procedures and distance of eye structures. 
Phaco - Emulsification Using Rotating Needle - A Newer Cheaper

Technique

Table 2: Change of temperature in the lens and surrounding water during emulsification of lens in a container

\begin{tabular}{|c|c|c|}
\hline Time (s) & Temp. of Water $\left({ }^{\circ} \mathrm{C}\right)$ & Temp of Lens $\left({ }^{\circ} \mathrm{C}\right)$ \\
\hline 00 & 28.2 & 29.5 \\
\hline 10 & 27.9 & 29.1 \\
\hline 20 & 27.9 & 28.2 \\
\hline 30 & 27.8 & 28.1 \\
\hline 40 & 27.7 & 28.1 \\
\hline 50 & 27.7 & 28.1 \\
\hline $1: 00$ & 27.6 & 28.0 \\
\hline $1: 10$ & 27.6 & 27.8 \\
\hline $1: 20$ & 27.6 & 27.9 \\
\hline $1: 30$ & 27.6 & 28.8 \\
\hline $1: 40$ & 27.8 & 27.9 \\
\hline $2: 00$ & 27.8 & 28.2 \\
\hline $2: 10$ & 27.8 & 28.1 \\
\hline $2: 20$ & 27.8 & 28.2 \\
\hline $2: 30$ & 27.8 & 28.2 \\
\hline $2: 40$ & 27.7 & 28.1 \\
\hline $2: 50$ & 27.9 & 28.4 \\
\hline 3:00 & 28.1 & 30.6 \\
\hline $3: 10$ & 28.2 & 28.8 \\
\hline
\end{tabular}

Table 3: Change of temperature in the lens during the operation in a small container

\begin{tabular}{|c|r|c|}
\hline Time $(\mathrm{s})$ & Temp of Lens ( $\left.{ }^{\circ} \mathrm{C}\right)$ \\
\hline 00 & 29.7 \\
\hline 05 & 29.6 \\
\hline 10 & 29 \\
\hline 15 & 29.1 \\
\hline 20 & 29.9 \\
\hline 25 & 27.9 \\
\hline 30 & 29.1 \\
\hline 35 & 29.1 \\
\hline 40 & 29.2 \\
\hline 45 & 29.2 \\
\hline 50 & 29.2 \\
\hline
\end{tabular}

Table 4: Change of temperature in the cornea and the anterior chamber during the operation on a goat eye

\begin{tabular}{|c|c|c|}
\hline Time $(\mathrm{s})$ & Temp. of Cornea $\left({ }^{\circ} \mathrm{C}\right)$ & Temp of Anterior Chamber $\left({ }^{\circ} \mathrm{C}\right)$ \\
\hline 00 & 29.8 & 28.8 \\
\hline 10 & 29.9 & 29.0 \\
\hline 20 & 29.9 & 29.2 \\
\hline 30 & 29.9 & 29.4 \\
\hline 40 & 29.9 & 29.6 \\
\hline 50 & 29.9 & 29.1 \\
\hline $1: 00$ & 29.9 & 28.9 \\
\hline $1: 10$ & 29.9 & 29.1 \\
\hline
\end{tabular}


Phaco - Emulsification Using Rotating Needle - A Newer Cheaper

Technique

Copyright:

(c) 2017 Yousuf T.

\begin{tabular}{|l|l|l|}
\hline $1: 20$ & 29.9 & 29.2 \\
\hline $1: 30$ & 29.9 & 29.4 \\
\hline $1: 40$ & 29.9 & 29.5 \\
\hline $1: 50$ & 29.9 & 29.7 \\
\hline $2: 00$ & 30.0 & 29.7 \\
\hline $2: 10$ & 30.0 & 29.8 \\
\hline $2: 20$ & 30.0 & 30.0 \\
\hline $2: 30$ & 30.0 & 29.8 \\
\hline $2: 40$ & 30.1 & 29.9 \\
\hline $3: 00$ & 30.1 & 30.0 \\
\hline
\end{tabular}

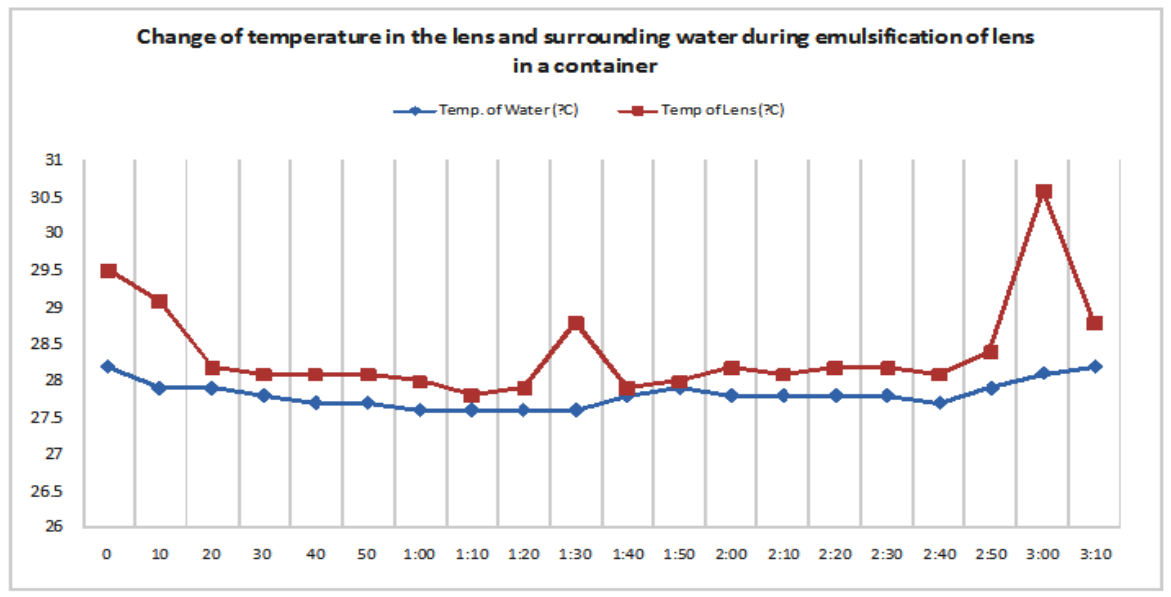

Figure 2: Change of temperature in the lens and surrounding water during emulsification of lens in a container

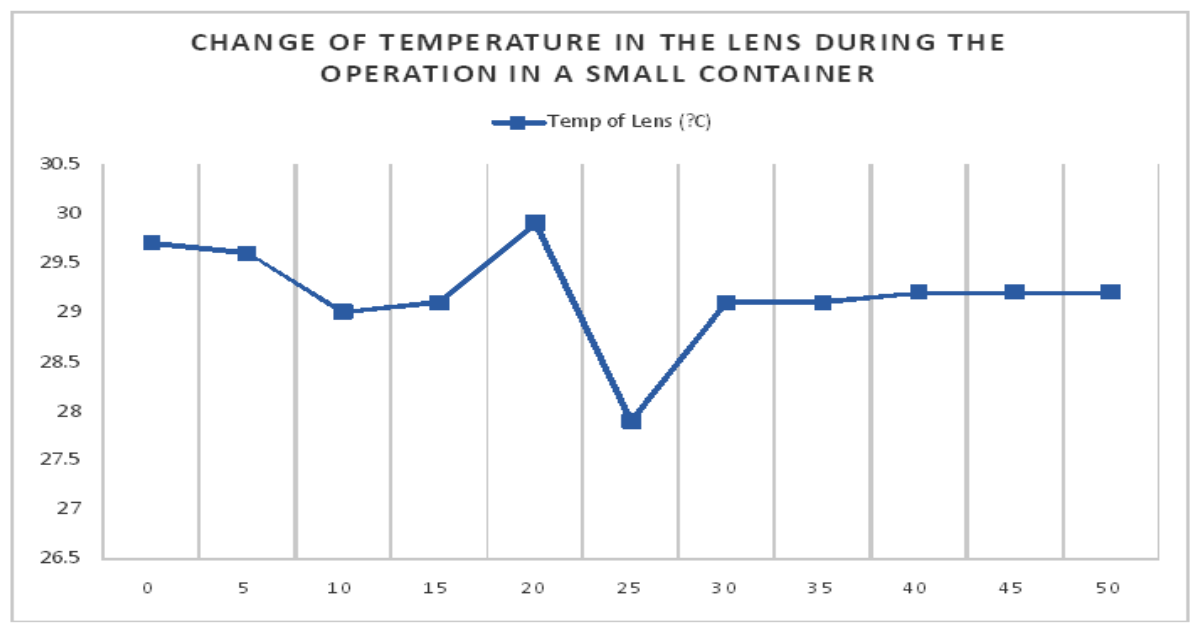

Figure 3: Change of temperature in the lens during the operation in a small container 


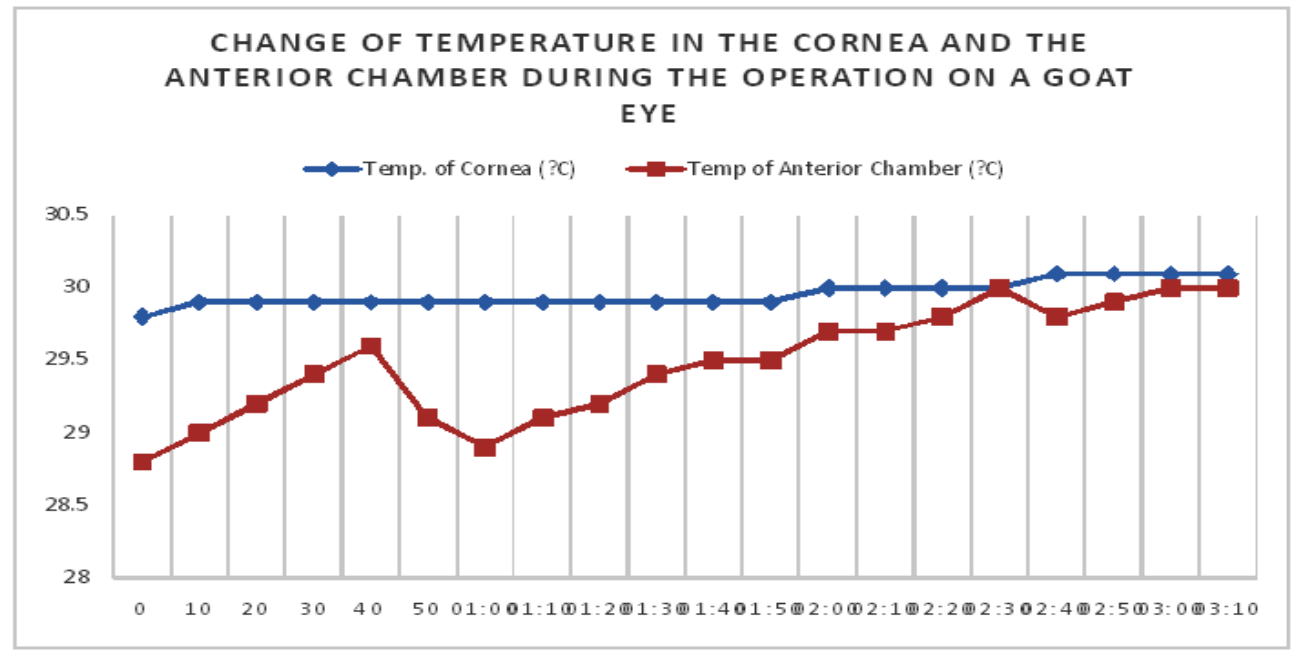

Figure 4: Change of temperature in the cornea and the anterior chamber during the operation on a goat eye

\section{Conclusion}

Phaco emulsification surgery for cataractous lens extraction is latest technique accepted worldwide where a titanium needle is vibrating with high frequency i.e. 28,000 to 40,000 hertz; vibrating spike is 6 to 15 micron by means of ultrasound. This device is very effective for extraction of cataract's lens, but the problem is this instrument is not a simple machine, during operation so many problems arise like changes of temperature, changes of pressure changes of vacuum etc so if surgeon is not skilled enough he may mistake causing serious vision threatening problem for patient.

But my device is a simple device; no raise much temperature to cause thermal injury to surrounding structure during operation. Efficacy is almost same to phaco machine. I have used mechanical energy to rotate a needle which will emulsify lens material successfully and lens fibers will convert into a jelly like substances and then it will evacuated by two way irrigation aspiration canola. So, by using my proposed device we want to decrease severe complications during surgery which will be cost effective as well. The basic mechanism of new device is different than phaco machine. Proposed device will work by rotating a special type of needle (Yousuf's needle) at the rate of less than 2,000 rpm where phaco machine works by vibrating a needle which is made of titanium. This low rotation can be achieved by using a 'DC' motor where voltage will be between1.5 to12 volts.

\section{References}

1. Charles D. kelman, MD phako, phakonit and laser phako- a Quest for the best (highlights of ophthalmology. 2002:1-8.)

2. Dada VK, Sindhu N. Cataract in enucleated goat eyes: training model for phacoemulsification. J Cataract Refract Surg. 2000;26(8):11141116.

3. Mohammadi SF, Mazouri A, Jabbarvand M, Rahman-A N, Mohammadi A. Sheep practice eye for ophthalmic surgery training in skills laboratory. J Cataract Refract Surg. 2011;37(6):987-991. doi: 10.1016/j. jcrs.2011.03.030

4. Van Vreeswijk, Pameyer JH. Inducing cataract in postmortem pig eyes for cataract surgery training purposes. J Cataract Refract Surg. 1998;24(1):17-18. 